\title{
A Study on Burnout of Nurses in the Period of COVID-19
}

\author{
Liu Guixia*, Zhang Hui \\ First Affiliated Hospital of Anhui Medical University, Hefei, China \\ Email address: \\ 386901459@qq.com (Liu Guixia),2761145197@qq.com (Zhang Hui) \\ ${ }^{*}$ Corresponding author
}

\section{To cite this article:}

Liu Guixia, Zhang Hui. A Study on Burnout of Nurses in the Period of COVID-19. Psychology and Behavioral Sciences. Vol. 9, No. 3, 2020, pp. 31-36. doi: 10.11648/j.pbs.20200903.12

Received: May 14, 2020; Accepted: June 4, 2020; Published: June 17, 2020

\begin{abstract}
Purpose: Because of the particularity of nursing work, clinical nurses have different degrees of burnout, which is related to various factors. The battle against COVID-19 is a huge challenge, and the burnout of nurses may not be as same as it used to be. This study examined the level of nurses' burnout and analyzed its influencing factors in the period of COVID-19. Methods: The questionnaire was released in the form of questionnaire star, and the level of burnout, anxiety and depression were measured by Maslach Burnout Inventory (MBI), Self-rating Anxiety Scale (SAS) and Self-rating Depression Scale (SDS), respectively. Results: Except that the dimension of personal accomplishment was negatively correlated with anxiety and depression $(\mathrm{r}=-0.243 \sim-0.408, \mathrm{P}<0.05)$, there were positive correlations between emotional exhaustion, depersonalization, total score of MBI and anxiety, depression $(\mathrm{r}=0.447 \sim 0.738, \mathrm{P}<0.01)$. Multiple stepwise regression analyses showed that burnout was positively correlated with anxiety $(\beta=0.688)$, and negatively with working years $(\beta=-5.597)$. Conclusions: The incidence of nurses' burnout was high in the period of COVID-19, which was closely related to anxiety and depression. When nurses were more anxious and had shorter working years, their burnout was more likely to be stronger. Therefore, both managers and nurses themselves should pay attention to the burnout. Nurses with symptoms of anxiety and shorter working time are the focus of attention and intervention.
\end{abstract}

Keywords: COVID-19, Burnout, Anxiety, Depression

\section{Introduction}

Burnout should be described as a dangerous condition of overwork that can lead to physical or mental illness, such as high blood pressure, tinnitus, or depression [1]. About 59.1\% of clinical nurses have some degree of burnout, the occurrence and severity of which are related to many factors, such as social, environmental and personal factors [2]. When individuals feel tired, they tend to take evasive behavior in the stressful situations, which can exacerbate stress responses and ultimately generate negative emotions, as well as irrational beliefs such as dependency, avoidance of problems, and helplessness [3]. The study found that burnout was directly related to cognitive and emotional symptoms, and emotional problems, especially anxiety and depression, were considered as risk factors for burnout [4]. Burnout has a significant correlation with anxiety and depression $[1,5,6]$, $77.1 \%$ of burnout is related to anxiety or depression, and $84.0 \%$ of anxiety and depression is related to burnout [7].
Coronavirus disease 2019 (COVID-19) is caused by severe acute respiratory syndrome coronavirus 2 (SARS-CoV-2). Since first reported in December 2019, it has captured the world's attention because of its similarity to MERS-CoV and SARS-CoV in leading to fatal respiratory disease, and its high transmission capability and mortality, as well as economic disruption [8, 9]. According to DXY. DX Doctor COVID-19 Global Pandemic Real-time Report (https://ncov.dxy.cn/ncovh5/view/pneumonia?from=singlemes sage $\&$ isappinstalled $=0 \& \mathrm{sf}=1 \& \mathrm{dn}=2$ ), as of 21 April 2020, over 200 countries/regions have reported 2446840 COVID-19 cases, of which 170993 (7.00\%) have died. COVID-19 has grown up to be a serious public health threat and global health emergency [10]. Up to date, we still do not have any effective and specific anti-SARS-CoV-2 drugs [10, 11]. In fact, it is not certain when it will end. The outbreak of COVID-19 has become a clinical threat to the healthcare workers as well as the general population around the world [11]. Front-line health care workers may have experienced conflict between their professional ethics, sense of responsibility and fear of being 
infected. As the direct caregiver of patients, nurses have more chances of close contact with them, greater risk of being infected, and double aggravation of physical and mental load. According to a survey of the first batch of clinical first-line support nurses in Hubei Province who fought against COVID$19,92.68 \%$ of them developed psychological problems within two weeks [12]. However, it is not clear how these problems affect nurses' burnout and whether they are exacerbated by anxiety and depression. Based on the cross-sectional investigation, present study investigated the changes of nurses' occupational emotion, sense of personal achievement and other related influencing factors in this period, so as to provide a new perspective and ideas for the subsequent intervention.

\section{Materials and Methods}

\subsection{Study Samples and Recruitment Procedure}

This manuscript was a cross sectional descriptive study on nurses' burnout during COVID - 19 and analyzed its influencing factors. Using convenient sampling method, the questionnaire was released in wechat client in the form of questionnaire star on February 2, 2020. Wechat friends working as nurses were invited to take part in the questionnaire star survey, reachable through a dedicated web link, and then to forward this web link to the other nurse friends of their hospital to provide them with the possibility of completing the investigation as well. The online survey included measures of burnout, anxiety, depression and a short sociodemographic questionnaire. To qualify for the study, being currently employed as a registered practical nurse was the sole criterion. Participation was voluntary and did not involve any compensation. The research proposal has been presented to the Institute's Ethics Committee.

A total of 92 practical nurses took part in the study $\left(\mathrm{M}_{\mathrm{AGE}}=\right.$ $30.60, \mathrm{SD}_{\mathrm{AGE}}=6.27 ; 91.30 \%$ female). The study sample mainly included Nurse Practitioner (43.50\%), Nurse (27.20\%), Nurse-in-charge (27.20\%) and Associate Professor of Nursing (2.20\%). Most participants were undergraduate $(87.00 \%)$, few was junior college student (5.40\%) and post-graduate (7.60\%). $79.35 \%$ of them reported that they had experience in nursing SARS/ Influenza A/Avian Influenza. Participants' mean length of employment was 9.00 years $(\mathrm{SD}=6.27)$. They were all from Grade III, Class A hospital in Anhui Province, China. Most of them $(63.04 \%)$ came from the general ward of non COVID-19, less than half being from COVID-19 ward (ICU: $13.04 \%$; general ward: $23.91 \%$ ).

\subsection{Study Instruments}

\subsubsection{Sociodemographic Questionnaire}

The sociodemographic questionnaire was devised by the researcher in order to obtain information about sociodemographic variables of the participants. It included questions such as gender, age, technical title, education level, sections or clinical units and number of years spent on the job, etc. It also contained whether the participants had experience in nursing SARS / influenza A / Avian Influenza.

\subsubsection{Maslach Burnout Inventory (MBI)}

MBI $[5,13]$ is a 22-item questionnaire, which is generally considered as a standard scale to measure burnout. It is also a Likert 7-point scale, with the scores ranging from 0 to 6 ( 0 : never occurs to me, 6 : it occurs to me every day). 22 questions include three dimensions: emotional exhaustion, depersonalization and personal accomplishment. The emotional exhaustion dimension mainly measures the emotional response caused by excessive work pressure, which is composed of items $1,2,3,6,8,13,14,16$ and 20 . The total score ranges from 0 to 54 points, 19 to 26 points are moderate burnout, and above 26 points are high burnout. The depersonalization dimension mainly measures the attitude and feeling of the respondents towards the service object under the pressure situation, which is composed of $5,10,11,15$ and 22 items, with a total score ranging from 0 to 30,6 to 9 indicating moderate burnout, and more than 9 for high burnout. The dimension of personal accomplishment consists of the remaining 4, 7, 9, 12, 17, 18, 19 and 21 items (all of which are scored in the reverse direction). The overall score is 0 to 48 , with a score of 34 to 39 indicating moderate burnout and a score below 34 meaning high burnout. According to the total score of MBI, burnout is divided into non-burnout (below 50), mild burnout (50 to 75 ), moderate burnout (76 to 100) and severe burnout (above 100). The scale has been widely used at home and abroad and has good reliability and validity.

\subsubsection{Self-rating Anxiety Scale (SAS)}

SAS [5] contains 20 items $(5,9,13,17,19$ reverse score), which mainly evaluate the anxiety symptoms of the investigators. Likert 5-point score is used in the scale, and the integral part of the total score multiplied by 1.25 is the standard score, which is normal if the standard score is less than 50, mild anxiety if 50 59, moderate anxiety if 60 69, and severe anxiety if it is more than 70. SAS has good reliability and validity.

\subsubsection{Self-rating Depression Scale (SDS)}

SDS $[5,14]$ is composed of 20 items $(2,5,6,11,12,14,16$, $17,18,20$ reverse score), which mainly measures the depression symptoms of the subjects. The scoring method of the scale is similar to that of SAS, with a standard score less than 53 as normal, 53 62 as mild depression, 63 72 as moderate depression, and more than 72 as severe depression. The scale also has good reliability and validity.

\subsection{Statistical Analysis Methods}

SPSS 17.0 was used for statistical analysis. Firstly, burnout, anxiety and depression were analyzed and compared with the norm in order to evaluate the status of burnout, anxiety and depression of nurses during COVID-19. Then single factor analyses were used to analyze the influence of anxiety and depression on burnout, as while as the impact of social demographic factors on burnout, anxiety and depression. After that, Spearman/Pearson correlation analyses explored the correlation between burnout and anxiety, depression and social demographic factors. The comorbidities between burnout and 
anxiety and depression were discussed by chi-square cross list method. Finally, multiple regression analyses were utilized to explore the influencing factors of nurses' burnout in COVID-19 period. $P$-values $\leq 0.05$ (double sided) were considered to have statistical significance, with $\alpha$ level set at 0.05 .

\section{Results}

\subsection{Nurses' Burnout, Anxiety and Depression During COVID-19}

During COVID-19, the prevalence of nurses' burnout was
$81.6 \%$, which was mainly mild. $43.5 \% \sim 62.0 \%$ of the respondents showed moderate to high burnout in depersonalization, emotional exhaustion and personal accomplishment. The incidence of anxiety was $41.3 \%$, including $29(31.5 \%)$ with mild anxiety, 7 (7.6\%) with moderate anxiety, and $2(2.2 \%)$ with severe anxiety. There were $27(29.3 \%)$ nurses with mild depression, 23 (25.0\%) ones with moderate depression, and $3(3.3 \%)$ ones with severe depression. The prevalence of depression was $57.6 \%$. Table 1 displays the scores of burnout, anxiety and depression and their comparison with the norm $[13,14]$.

Table 1. The score of burnout, anxiety and depression and their comparison with the norm.

\begin{tabular}{|c|c|c|c|c|c|c|c|}
\hline \multirow{2}{*}{\multicolumn{2}{|c|}{ Variables }} & \multicolumn{3}{|l|}{ This study } & \multirow[b]{2}{*}{ The norm } & \multirow[b]{2}{*}{$T$-value } & \multirow[b]{2}{*}{$P$-value } \\
\hline & & Minimum value & Maximum value & $\bar{x} \pm s$ & & & \\
\hline \multirow{4}{*}{ MBI } & $\mathrm{EE}$ & 0 & 49 & $19.2 \pm 10.96$ & $22.19 \pm 9.53$ & -2.611 & 0.011 \\
\hline & DP & 0 & 19 & $5.78 \pm 4.36$ & $7.12 \pm 5.22$ & -2.944 & 0.004 \\
\hline & PA & 0 & 48 & $34.45 \pm 9.84$ & $36.53 \pm 7.34$ & -2.010 & 0.047 \\
\hline & Total score & 0 & 95 & $59.46 \pm 14.66$ & - & & \\
\hline \multicolumn{2}{|l|}{ SAS } & 25 & 73 & $46.89 \pm 10.47$ & $37.23 \pm 12.58$ & 15.072 & 0.000 \\
\hline \multicolumn{2}{|l|}{ SDS } & 27 & 78 & $50.7 \pm 12.11$ & $41.88 \pm 10.75$ & 7.019 & 0.000 \\
\hline
\end{tabular}

Note:

1. MBI: Maslach Burnout Inventory; EE: emotional exhaustion; DP: depersonalization; PA: personal accomplishment. SAS: Self-rating Anxiety Scale; SDS: Self-rating Depression Scale.

2. The norm of MBI: $n=1104$, The norm of SAS: $n=1158$, The norm of SDS: $n=1340$.

\subsection{Single Factor Analyses of Nurses' Burnout in COVID-19 Period}

Through single factor analyses, burnout, anxiety and depression of nurses in COVID-19 period were not statistically significant in age, gender, years of service, technical title, educational background, sections or clinical units and nursing experience of SARS / influenza A / Avian Influenza $(P \geq 0.05)$. However, burnout had statistical significance in anxiety and depression $(P<0.05)$. Table 2 and table 3 respectively present the results of univariate analyses of nurses' burnout in depression and anxiety in COVID-19 period.

Table 2. Single factor analyses of burnout in depression degree.

\begin{tabular}{|c|c|c|c|c|c|}
\hline Depression degree & $\mathbf{n}$ & $\mathbf{E E}$ & DP & PA & Total score of MBI \\
\hline Normal & 39 & $12.23 \pm 6.92$ & $3.92 \pm 3.55$ & $39.23 \pm 10.84$ & $55.38 \pm 14.74$ \\
\hline Mild depression & 27 & $18.59 \pm 7.69$ & $5.48 \pm 3.48$ & $31.78 \pm 7.87$ & $55.85 \pm 10.45$ \\
\hline Moderate Depression & 23 & $29.30 \pm 9.72$ & $8.04 \pm 4.16$ & $30.96 \pm 6.66$ & $68.30 \pm 13.98$ \\
\hline Severe depression & 3 & $38.00 \pm 9.54$ & $15.33 \pm 3.51$ & $23.67 \pm 4.93$ & $77.00 \pm 15.71$ \\
\hline$F$-value & & 27.748 & 12.929 & 7.077 & 6.873 \\
\hline$P$-value & & 0.000 & 0.000 & 0.000 & 0.000 \\
\hline
\end{tabular}

Table 3. Single factor analyses of burnout in anxiety degree.

\begin{tabular}{|c|c|c|c|c|c|}
\hline Anxiety degree & $\mathbf{n}$ & $\mathbf{E E}$ & DP & PA & Total score of MBI \\
\hline Normal & 54 & $14.81 \pm 9.21$ & $4.67 \pm 3.71$ & $36.57 \pm 10.86$ & $56.06 \pm 14.46$ \\
\hline Mild anxiety & 29 & $22.79 \pm 9.21$ & $6.24 \pm 3.88$ & $31.97 \pm 7.00$ & $61.00 \pm 12.30$ \\
\hline Moderate anxiety & 7 & $32.00 \pm 8.37$ & $9.29 \pm 5.09$ & $32.00 \pm 8.25$ & $73.29 \pm 12.59$ \\
\hline Severe anxiety & 2 & $41.00 \pm 11.31$ & $17.00 \pm 2.82$ & $23.67 \pm 4.93$ & $80.50 \pm 20.51$ \\
\hline$F$-value & & 13.879 & 9.154 & 2.728 & 5.13 \\
\hline$P$-value & & 0.000 & 0.000 & 0.049 & 0.000 \\
\hline
\end{tabular}

\subsection{Correlation Analyses of Nurses' Burnout in COVID-19 Period}

By Spearman correlation analyses, burnout, anxiety and depression of nurses in COVID-19 period were not statistically significant in age, gender, years of service, technical title, educational background, sections or clinical units and nursing experience of SARS / influenza A / Avian Influenza $(P \geq 0.05)$. However, burnout had statistical significance in anxiety and depression $(P<0.05)$. According to Pearson correlation analyses, except for the negative correlation between personal accomplishment and anxiety and depression $(r=-0.243 \sim-0.408, P<0.05)$, there were positive correlations between emotional exhaustion, depersonalization, total score of MBI and anxiety and depression $(r=0.447 \sim 0.738$, 
$P<0.01)$. The results are shown in table 4 .

Table 4. Correlations between burnout and anxiety and depression.

\begin{tabular}{lllllll}
\hline Variables & SDS & SAS & EE & DP & PA & Total score of MBI \\
\hline SDS & - & & & & \\
SAS & $0.797^{* *}$ & - & - & & \\
EE & $0.738^{* *}$ & $0.673^{* *}$ & - & - & \\
DP & $0.569^{* *}$ & $0.490^{* *}$ & $0.701^{* *}$ & $-0.320^{* *}$ & - & \\
PA & $-0.408^{* *}$ & $-0.243^{*}$ & $-0.278^{* *}$ & $0.605^{* *}$ & $0.368^{* *}$ & - \\
Total score of MBI & $0.447^{* *}$ & $0.485^{* *}$ & $0.768^{* *}$ & 0.00 \\
\hline
\end{tabular}

Note: **. Significant correlation was found at the level of 0.01 (bilateral);

*. Significant correlation was found at the level of 0.05 (bilateral);

MBI: Maslach Burnout Inventory; EE: emotional exhaustion; DP: depersonalization; PA: personal accomplishment. SAS: Self-rating Anxiety Scale; SDS: Self-rating Depression Scale.

\subsection{Analyses of the Comorbid Relationship of Burnout, Anxiety and Depression of Nurses in COVID-19 Period}

The chi-square cross list method was used to explore the comorbid relationship between nurses' burnout and anxiety and depression. The results showed that $86.84 \%$ (33/38) and $83.02 \%(44 / 53)$ of the nurses with anxiety and depression were likely to suffer from burnout, while $44.00 \%$ (33/75) and $58.67 \%(44 / 75)$ of ones with burnout were likely to have anxiety and depression, respectively.

\subsection{Multiple Regression Analyses of Nurses' Burnout in COVID-19 Period}

Multiple stepwise regression analyses were used to explore the influencing factors of burnout. The total score of burnout was taken as the dependent variable, and the independent variables were age $(<25$ years $=1,25-35$ years $=2,>35$ years $=3)$, gender $($ male $=1$, female $=2)$, working years $(<2$ years $=1,2 \sim 5$ years $=2,6 \sim 10$ years $=3,11 \sim 20$ years $=4$, $>$ 20 years $=5$ ), technical title (nurse $=1$, nurse practitioner $=2$, nurse-in-charge $=3$, associate professor of nursing $=4$ ) 、 educational background (junior college student $=1$, undergraduate $=2$, post-graduate $=3$ ) 、 experience in nursing SARS/ Influenza A/Avian Influenza (no=1, yes=2), sections or clinical units (COVID-19 common ward =1, COVID-19 intensive care ward $=2$, common ward $=3$ ) and anxiety and depression (original score). The results showed that anxiety and working years together accounted for $26.8 \%$ of the variation in burnout, as showed in table 5 .

Table 5. Multiple regression analyses of burnout.

\begin{tabular}{lllllll}
\hline Independent variables & $\mathbf{B}$ & $\boldsymbol{S E}$ & Beta & $\boldsymbol{t}$ & $\boldsymbol{P}$ & $\boldsymbol{R}^{\mathbf{2}}$ \\
\hline SAS & 0.688 & 0.127 & 0.491 & 5.409 & 0.000 & \multirow{2}{*}{0.268} \\
Working years & -5.597 & 2.802 & -0.181 & -1.997 & 0.049 & \multirow{2}{*}{0.252} \\
\hline
\end{tabular}

Note: SAS: Self-rating Anxiety Scale, $F=16.297, P=0.000$.

\section{Discussion}

The burnout incidence of nurses in COVID-19 period was $81.6 \%$, which was slightly lower than ICU nurses (89.57\%), but significantly higher than that of general ward ones (49.15\%) [5]. Compared with the norm [13], there was statistical significance in the three dimensions of burnout. On the one hand, it shows that although the physical and mental load of nurses in COVID-19 period was increased, they had high morale and work enthusiasm, full of empathy for patients, and being able to think from the perspective of patients with empathy, so their depersonalization characteristics were not obvious. On the other hand, because of the serious epidemic situation and the lack of specific drug treatment, what the nurse can do seemed to be limited, compared with the treatment effect, so the sense of theirs personal accomplishment was low.

Epidemiological studies have shown that about $17 \sim 41 \%$ of nurses have depressive symptoms, and nearly $20 \sim 49 \%$ of ones have anxiety symptoms in different countries [3]. In this study, the results indicated that the incidence of anxiety was similar to the former, but the one of depression was significantly higher than the previous study. There were significant correlations between burnout and anxiety and depression. With the aggravation of anxiety and depression, the score of burnout gradually increased, the characteristics of emotional exhaustion and depersonalization were obvious, the sense of personal accomplishment was reduced, and the probability of burnout was high in the nurses with anxiety and depression, which was similar to the results of Hardy P et al, but the comorbidity between burnout and anxiety and depression was lower than the latter [7]. Regression analyses showed that anxiety and years of service together accounted for $26.8 \%$ of the variation in burnout. Above results suggested that there was a certain degree of anxiety and depression in nurses in COVID-19 period, especially depression, but anxiety had a greater impact on burnout, and with the length of service, the burnout tended to decrease.

Similar to poor resilience and healthy life belief, high intensity and long-term stress may increase the risk of depression, anxiety and other psychological disorders of nurses [3]. Nursing is an ever so stressful and challenging profession due to the constant contact with patients, being responsibility for human health, carrying out clinical 
operations, dealing with dead patients, responding quickly to urgent and unpredictable situations, excessive workplace noise and year-round rotation [15]. From an etiological point of view, unresolved stress, which is thought to play an important role in burnout, plays a crucial role in the development of depression, and there is strong evidence that, depressive symptoms are a basic response to unresolved stress [16]. Depression, anxiety and stress are important psychological problems that may cause emotional problems, mental fatigue and hinder mental health, and are also the basis of burnout [15]. Anxiety and depression overlap with burnout, and anxiety is a key aspect of burnout [16]. In turn, burnout may enlarge this negative emotion. When emotional exhaustion is more obvious, work pressure has a greater impact on depression and anxiety [3], further deepening or affecting the occurrence and development of anxiety and depression, and may also make burnout more serious. Burnout is not only the existence of negative emotions, but also the absence of positive emotions. In burnout, "positive emotions erode their ability to counteract negative emotions", and people who experience burnout first feel "helpless, hopeless, and powerless", usually displaying "feelings of sadness, tears, and depression" [16].

Nurses are known to be at high risk of burnout for a variety of reasons, such as the rigor of patient care [15]. There were significant positive correlations between psychological symptoms (depression, anxiety, and stress) and all dimensions of burnout. In other words, the more serious the psychological symptoms (depression, anxiety and perceived stress), the higher the level of burnout [15], which is consistent with the current research results. Nurses' burnout may have a serious impact on their mental health. Self-control and emotional regulation can effectively and quickly solve problems and dissolve anxiety [6]. Some measures, such as seeking support from friends, colleagues, supervisors and specific mental health professionals, can help prevent and ease burnout $[3,6]$. In addition, good rest, reasonable nutrition, proper physical exercise, personal interests and hobbies, and timely vacation when feeling overtired can also protect employees from the impact of chronic stress and prevent the occurrence of burnout $[3,6]$. In view of the occupational burnout of nurses in COVID-19 period, clinical intervention can be conducted from this perspective.

This study also found that although age, gender, technical title, educational background, nursing experience of SARS/ Influenza A/Avian Influenza and sections or clinical units had little impact on nurses' burnout in COVID-19 period, there was a statistical significance in years of service. This suggests that in this special period, all the people are on the alert and ready to fight against COVID-19. In the face of such a severe epidemic situation, whether they are male or female, how old they are, no matter where they come from, whether they have clinical nursing experience of serious respiratory disease such as SARS, most nurses have similar psychology, but many years of clinical experience had made them learn to control more effectively and timely deal with negative emotions to protect themselves from emotional exhaustion and dehumanization [17], thereby reducing the burnout.

\section{Conclusions}

To sum up, the incidence of nurses' burnout in COVID-19 period was high, which was correlated with anxiety and depression. The mental health problems of nurse should not be underestimated. Both managers and nurses themselves should pay attention to the burnout. Nurses with symptoms of anxiety and shorter working time are the focus of attention and intervention. However, since the current study was conducted in the early stage of COVID-19 occurrence and the samples were from the same region, the results need to be confirmed by further research. In addition, the psychology of caregivers varies in different regions and periods of illness, and people's psychological changes are also dynamic. Therefore, in the future, dynamic research in depth of a large sample should be carried out at different multicenter and period of illness to provide references for the subsequent clinical development of appropriate intervention measures.

\section{Acknowledgements}

Special thanks to all the nursing colleagues who participated in this survey. Thank for your support and cooperation. It is your courage and bravery, regardless of personal gains and losses, and your spirit of trying your best to pay that deeply moved and supported me to complete this manuscript.

\section{Conflict of Interest}

All the authors do not have any possible conflicts of interest, including financial interests or relationships.

\section{References}

[1] Schwarzkopf K, Straus D, Porschke H, Znoj H, von Känel R (2019). Is it all about Depression? Determinants of Burnout among Employees Referred for Inpatient Treatment of Job-Related Stress Disorders. Z Psychosom Med Psychother 65, 183-197.

[2] Wei-bin Z, Qiu-huan DU (2019). The correlation between depression and job burnout in standardized training of resident physicians and its influencing factors. Journal of Harbin Medical University 53, 94-97. DOI: 10.3969/j.issn.1000-1905.2019.01.022.

[3] Chen J, Li J, Cao B, Wang F, Luo L, Xu J (2020). Mediating effects of self-efficacy, coping, burnout, and social support between job stress and mental health among young Chinese nurses. J Adv Nurs 76, 163-173.

[4] Yaoyao S, Fei L, Fenglin C (2019). Job burnout subtypes and emotional problems among medical staff in a tertiary hospital. Journal of Shandong University (Health Science) 57, 100-105. DOI: $10.6040 /$ j.issn.1671-7554.0.2018.1488. 
[5] Yanyan Z, Tingting Z, Bo LI (2018). A related study of occupational burnout and depression, anxiety in nurses working at Intensive Care Unit. Journal of Psychiatry 31, 442-445. DOI: 10.3969/j.issn.2095-9346.2018.06.011.

[6] Papathanasiou IV, Tsaras K, Kleisiaris CF, Fradelos EC, Tsaloglidou A, Damigos D (2017). Anxiety and Depression in Staff of Mental Units: The Role of Burnout. Adv Exp Med Biol 987, 185-197.

[7] Hardy P, Costemale-Lacoste JF, Trichard C, et al (2019). Comparison of burnout, anxiety and depressive syndromes in hospital psychiatrists and other physicians: Results from the ESTEM study. Psychiatry Res 112662.

[8] Zhou YH, Qin YY, Lu YQ, et al (2020). Effectiveness of glucocorticoid therapy in patients with severe novel coronavirus pneumonia: protocol of a randomized controlled trial. Chin Med J (Engl), 2020. DOI: 10.1097/CM9.0000000000000791.

[9] Zhao D, Yao F, Wang L, et al (2020). A comparative study on the clinical features of COVID-19 pneumonia to other pneumonias. Clin Infect Dis, 2020. DOI: 10.1093/cid/ciaa247.

[10] Yuen KS, Ye ZW, Fung SY, et al (2020). SARS-CoV-2 and COVID-19: The most important research questions. Cell Biosc 10, 40. DOI: 10.1186/s13578-020-00404-4.

[11] Lai CC, Shih TP, Ko WC, et al (2020). Severe acute respiratory syndrome coronavirus 2 (SARS-CoV-2) and coronavirus disease-2019 (COVID-19): The epidemic and the challenges. Int $\mathrm{J}$ Antimicrob Agents 55, 105924. DOI: 10.1016/j.ijantimicag.2020.105924.

[12] Mingchuan XU, Yue Z (2020). Psychological status survey of first clinical first?line support nurses fighting against pneumonia caused by a 2019 novel coronavirus infection. Chinese Nursing Research 34, 368-370. DOI: 10.12102/j.issn.1009-6493.2020.03.002.

[13] Lin MY (2009). Research on the correlation between burnout and personality among ICU nurses. Dissertation, Southern Medical University.

[14] Wang WJ, LU XF, SUN H, LIU Y, ZHAO YY, SU T (2019). Anxiety, depression and related factors in lung cancer inpatients. Academic Journal of Second Military Medical University $4 \quad 0, \quad 444-447 . \quad$ DOI: 10.16781/j.0258-879x.2019.04.0444.

[15] Sheikholeslami SVMRISAAHK (2017). The Relationship between Burnout Dimensions and Psychological Symptoms (Depression, Anxiety and Stress) Among Nurses. Journal of Holistic Nursing and Midwifery 27, 37-43.

[16] RENZO BIANCHI, IRVIN SAM S (2018). Personality and Social Psychology Burnout-depression overlap: Nomological network examination and factor-analytic approach. Scand J Psychol 59, 532-539. DOI: 10.1111/sjop.12460.

[17] Molassiotis A, Haberman M (1996). Evaluation of burnout and job satisfaction in marrow transplant nurses. Cancer Nurs $19,360-7$ 